\title{
Towards a gender-sensitive university
}

\author{
Rita Bencivenga and Eileen Drew
}

\section{Introduction}

One question underlies all the chapters of this book: Is the idea of a gendersensitive university a contradiction in terms? That universities are gendered institutions is not in dispute. Contributors to this book have demonstrated where the main problems lie; what needs to be done; and provided examples of good practice and change processes that would support the alignment of universities with gender sensitivity.

\section{Towards gender sensitivity: Identifying barriers, hurdles and glass ceilings in academia}

The diversity of barriers and mechanisms of resistance to change are evident from the succession of studies and reports, policy initiatives and interventions across the EU to promote gender equality and diversity in universities (Chapter 1). The shift towards a greater degree of gender equality has to be viewed against a political climate that exhibits often contradictory phenomena such as the emergence of neoliberal movements and new constructions of patriarchy, designed to limit and counter feminist calls for the engagement of all genders in the promotion of gender equality in academia (Chapter 2).

Work by Nielsen (Chapter 3), shows how the assumption of 'selection on merit' (developed further in Chapter 9) is circumvented by more than unconscious bias on the part of individual selectors and panels in the recruitment process. Recruitment is underpinned by the prevalence of practices that undermine the excellence principle, where expediency, cloaked as rationality and pragmatism, leads to preselection in determining the appointment of candidates to academic and research entry posts and professorial chairs. Chapter 3 considers the social and organisational contexts in which gender dynamics play out in recruitment and selection, focusing on the interaction of the decoupling strategies used by decision-makers in recruitment and selection. Nielsen's study shows how cultural and institutional environments shape most hiring decisions in academia, as gender bias systematically assumes the form of homophily and social closure in the networks of academic recruiters. 
Further evidence of the distortion of career progression on merit is posited in Chapter 4 by Kinahan, Dunne and Cahill, that illustrates the association of academic success with a male norm. Bias plays a role in assessment procedures involved in academic promotion, leading to a gendered perception of who is worthy of promotion and leadership. Workload allocation also contributes to the gender imbalance, allowing men to concentrate on their research and teaching, while female staff pick up the labour intensive administrative and pastoral work. As Pine testifies, from her personal and professorial experience:

Women are judged when they are not likeable enough. But being likeable, for all its social desirability, held us back at work. We ended up so busy doing all the pastoral care, and all the boring paperwork, and all the millions of unwanted jobs, that we never seemed to have time to ask for recognition.

$(2018,193)$

Chapters 3 and 4 underline the lack of fairness between male and female aspiring academics, in terms of recruitment, promotion, pay, mentorship and uneven workload allocation. They demonstrate that isolated interventions, such as unconscious bias training for recruitment and promotion panels or overhauling HR practices are not enough to overcome the deep-seated and endemic sexist practices that prevail in academia.

Work-life (im)balance is another barrier that working mothers face in academia. The irregular and informal academic work regime can be an important attraction for men and women embarking on an academic career since it appears to offer flexibility and autonomy. However, universities have undergone restructuring and downsizing as a consequence of budgetary cuts, hence working excessive hours has become the norm (Drew and Marshall, Chapter 5). While entrants to academic institutions may have expectations of flexible working, which is perceived as more compatible with becoming a parent or carer and achieving work-life balance, they quickly discover that they are part of 'greedy institutions' in which the career ladder to academic success is based on the capacity of academic 'sprinters'. A number of factors contribute to this: citations and rankings (Chapters 3 and 8); applying for research grants (Chapter 2); gaining visibility and international exposure through the conference circuit (Chapters 8 and 13); being career mobile and what Pine calls 'putting your research out there' $(2018,190)$. As a result, the academic race can be a very lonely, individualistic and predominantly male activity and one that undervalues even fundamentally human attributes: 'the qualities I generally associate with motherhood-love and support, empathy and nurturing - are not those I associate with being successful at work [as an academic in an Irish university]' (Pine 2018, 193).

The \#MeToo movement has served to lift the lid on what had previously been a closet topic of a spectrum of sexist behaviour including sexual harassment and violence. Pine $(2018,184)$ testifies to 'how often I encounter casual sexism, which for all its superficiality, can be bruising'. Sexual harassment and violence are now 
recognised as a critical barrier to gender equality in academia, particularly when the university campus presents itself as a physically dangerous setting to women. In Chapter 6, Paoletti, Quintin, Gray-Sadran and Squarcioni present the results of an institution-level initiative to identify the places on one French university campus where women felt unsafe and the actions, leading to remedies, that took place in response.

\section{Towards gender sensitivity: Addressing gender inequality in academia}

At a societal level, the pay gap between men and women is another barrier to gender equality in academia. In Chapter 7, Galligan, McMahon and Millar present the contexts of two universities: Trinity College, Dublin (TCD) and Queen's University, Belfast (QUB), prior to gender pay gap reporting, to compare the diverse results of a gender pay audit. The audit results demonstrate the impact of different national policies and sectoral employment conditions. In QUB, market conditions determine that there can be a wide variation between professorial salaries, starting at time of appointment. In TCD, a more structured and centralised approach to pay determination is the norm, allowing much less latitude for wage variance in the Irish higher education system. From the chapter's analysis, pay progression processes hold important lessons for the academic sector throughout Europe.

Obstacles and resistance to fairness in gender relations in academia are not only circumscribed to male and female academics; they also apply to the male category of gender itself. As any critical and intersectional approach would underline, men are not a monolithic group. Contending that gender equality in academia remains silent regarding men and masculinities, in Chapter 8 , Hearn draws upon: contemporary critical theorising, research and debates on men and masculinities, as well as of the situation of gendered individuals, men's individual academic identities and men's gendered careers in academia. Hearn demonstrates the variety of masculinities which have been observed in society and that can be used by policymakers, even at institution level, to drive a broader inclusion taking into account gender variations and intersectionality.

Like other private and public sector organisations and in society, unconscious bias is one of the main barriers to equality in academia. By playing a role in recruitment, promotion and funding decisions, it challenges the prevailing meritocratic principles underpinning academia. Gvozdanović and Bailey, in Chapter 9, show that it is important to adopt a comprehensive approach in designing a university-level policy to tackle gender inequalities. While unconscious bias plays an instrumental role in academic procedures that impact decisions and career outcomes, it is important to note that bias is not the only factor at play in those assessment procedures.

Implementing structural change towards gender equality involves several components: allies within and outside the organisation, change agents committed to their vision and strong enough to have a pervasive influence, funding to cover 
the costs of the process, aligning gender equality with the institution's objectives, being familiar with the relevant contexts internal and external, to drive organisational change. The SAGE Change Management Model promoted by Bailey and Drew (Chapter 10) takes account of this and the fact that academic institutions have different starting points. However, the process is similar, as is the need to deal with potential resistance. Drawing upon the evidence, communicating the need for action through raising awareness of the concepts and highlighting successes, are fundamental to the process of change towards gender equality in academia.

Promoting different concepts of leadership, to counter the challenges to gender equality, is the focus of Chapter 11 (Power). Leadership style is another gendered aspect of academia, influenced by the leader's environment, their situation and/or their relationship with followers. The powerful role of academic leadership in mitigating the effects of unconscious bias is also emphasised in Chapter 9 (Gvozdanović and Bailey) and the SAGE Change Management Model further underlines the need for top-down buy-in on the part of leaders and senior managers. The under-representation of women in senior leadership positions cannot be overstated and is reinforced by Faniko et al who suggest that:

the biggest challenge for diversity management may be to address the reluctance of women at the early stage of their career to value or express their own career commitment. Hence, efforts to enhance the representation of women in managerial positions should take into account the ambition-depressing effects for women of perpetuating a masculine organizational culture, especially at early career stages.

This finding supports the views expressed by Kinahan, Dunne and Cahill in Chapter 4 and the message by Husu in Chapter 13, that junior women academics deserve more acknowledgement, recognition and career mentoring to be able to progress as potential academic leaders.

Drawing upon Irish experience, Chapter 12 highlights the importance of using research and university funding as a lever in driving gender equality. Doona shows that the entry of the Athena SWAN Charter, a flagship accreditation scheme, has provided an essential impetus for institutions to address the issue of gender inequality. However, it was the political intervention to link institutional funding and research to the attainment of Athena SWAN that has radically transformed the sector over a short period of time. As Doona shows, all the Irish funding agencies have insisted on institutions producing and implementing Gender Equality Plans making gender equality a strategic goal.

In Chapter 13 Husu sets out the 'invisible', but all too damaging, effects of 'non-events' that are encountered throughout women's careers. In themselves, they may not represent major hurdles but cumulatively, the evidence shows that they contribute to the chilly climate and put a break on women's academic career 
advancement. This analysis is particularly important in explaining the higher drop-out of women at doctoral and postdoctoral levels throughout the EU (Chapter 1). Apart from the intense personal loss that this represents to the individual researcher or academic it is a major leakage of talent from the EU academic pool and is not sustainable.

\section{The role of GEPs in driving gender sensitivity}

A recurrent theme in all the book chapters is that concerted action is necessary to achieve more gender-sensitive academic institutions. Fixing the numbers and fixing the women has made way for fixing the institutions and fixing the knowledge base. The refinement and tailoring of a template for Gender Equality Plans, in the context of each institution, is one example of success and is a key mechanism in propounding this. GEPs depend on contextual and institutional factors along with the assessment of needs. They cover a wide range of thematic areas: leadership and decision-making, organisational culture, work-life balance, recruitment and career progression, gender in research and education practice and harassment and assault. All of these areas are core to redressing gender inequality and opening up educational opportunities for all genders.

\section{Conclusion}

Numerous challenges counteract with progress towards gender sensitivity. Prevailing forces that pose obstacles to driving a gender-sensitive university include: the emergence of far-right movements that seek to dismantle the hard-won advances in gender equality; a resurgence of patriarchy in new forms; and neoliberal managerialism that promotes a market-driven climate in which performativity, competitiveness and commodification prevail. Changes supporting the process towards equality and diversity require the increased engagement of men and the shift towards fluidity in fields previously excluded, such as gender, race, ethnicity, gender identity and expression and body ability.

Gender equality and gender-sensitivity are not fixed concepts but depend on context, previous experience, cultural and social traditions. They are affected by many factors, including globalisation, technology and ideologies. Becoming a gender-sensitive university involves a profound transformation by pursuing a multilevel, paradigmatic cultural shift, requiring motivation and resources. This book shows how awareness about gender equality and gender sensitivity are progressing in contemporary academia, while acknowledging the difficulties and obstacles in reaching those goals, drawing upon international and national contexts. The concept of a gender-sensitive university will be constantly evolving in response to progress achieved and the outstanding needs of those working or studying in it. It will be engaged with knowledge acquisition, drawing upon gendered learning and contributing to it. Progress will require re-envisioning academia to reflect a more inclusive conceptualisation of gender and intersectionality. 
Countervailing forces, that include globalisation, technological change and neoliberal ideologies, already impinge upon modern academic life, speeding up and intensifying work. These and other international and transnational trends are of growing importance in determining what is valued and who will progress to leadership positions in our institutions. Hence, gender equality constantly produces and reproduces backlash and resistance in ever more diverse forms. Heilman and Okimoto (2007) noted that it is women who face negative consequences, in traditional male domains, whether they act in a gender typical or atypical way, in conforming to, or rejecting, male norms of behaviour (Chapter 4).

As Hearn (in Chapter 8) and Drew and Marshall (in Chapter 5) point out, men, as well as women, may experience unconscious bias when they do not align with the requisites for the dominant work-centric-masculinist model. Gender-sensitive institutions must therefore promote new ways of organising and distributing fairly not only academic work but also responsibilities for family care and leadership. This will require changes in personal and collective behaviours; formal and informal norms, rules and behaviours. Academia will only be truly gender-sensitive if, learning from the past, it can avoid repeating the same mistakes, through the capability and the willingness to foresee and prevent new exclusions and new biases, implicit or otherwise. The future challenge will be to 'fix it forward' in all areas.

At a recent Irish Universities Association event, former Irish Minister of State for Higher Education Mitchell O'Connor (2019) shared a vision that encompasses the underlying message of this book:

There is enormous potential . . . to partner with European counterparts to expand the horizons of knowledge, enhance collaboration across boundaries and foster cultural understanding and experiences ... [to] promote common European values and a strengthened European identity by bringing together a new generation of Europeans, who are able to cooperate and work within different European and global cultures, in different languages, and across borders, sectors and academic disciplines. These alliances aim to act as models of good practice to progressively increase the quality, international competitiveness and attractiveness of European higher education.

\section{References}

Faniko, K., Ellemers, N. and Derks, B. (2016) Queen bees and alpha males: Are successful women more competitive than successful men? European Journal of Social Psychology, 46, 903-913.

Heilman, M. and Okimoto, T. (2007) Why are women penalized for success at male tasks? The implied communality deficit, Journal of Applied Psychology, 92 (1), 81-92.

Mitchell O'Connor, M. (2019) Speech at close of IUA Seminar on Ireland's Place in a new European University System, 29 January 2019, Dublin. Available at: https://www.educa tion.ie/en/Press-Events/Press-Releases/2019-press-releases/PR19-01-29.html

Pine, E. (2018) Notes to self, Penguin, London. 